\title{
Non-planar calibration phantoms for optical coherence tomography
}

Yang Lu, Neil Gordon, David Robinson, Ben Coldrick, Vladimir Mezentsev, et al.

Yang Lu, Neil Gordon, David Robinson, Ben Coldrick, Vladimir Mezentsev, Francesco Menduni, Antonio Fratini, Kate Sugden, "Non-planar calibration phantoms for optical coherence tomography," Proc. SPIE 10544, Advanced Fabrication Technologies for Micro/Nano Optics and Photonics XI, 105441B (22 February 2018); doi: 10.1117/12.2290422

SPE. Event: SPIE OPTO, 2018, San Francisco, California, United States 


\title{
Non-planar calibration phantoms for optical coherence tomography
}

\author{
Yang Lu*a ${ }^{\mathrm{a}}$, Neil Gordon ${ }^{\mathrm{a}}$, David Robinson ${ }^{\mathrm{b}}$, Benjamin Coldrick ${ }^{\mathrm{c}}$, Vladimir Mezentsev ${ }^{\mathrm{a}}$, Francesco \\ Menduni $^{\mathrm{d}}$, Antonio Fratini ${ }^{\mathrm{d}}$, Kate Sugden ${ }^{\mathrm{a}}$ \\ ${ }^{a}$ Aston Institute of Photonic Technologies, Aston University, Birmingham, B4 7ET, UK; \\ ${ }^{\mathrm{b}}$ Arden Photonics Ltd., Royston House, 267 Cranmore Boulevard, Solihull, B90 4QT, UK; \\ 'Optimec Limited, Unit B3, The Haysfield, Spring Lane North, Malvern, WR14 1GF, UK; \\ ${ }^{\mathrm{d}}$ Biomedical Engineering, Aston University, Birmingham, B4 7ET, UK
}

\begin{abstract}
Optical coherence tomography (OCT) is increasingly used in areas such as ophthalmology and contact lens metrology. However, in such cases, image distortion can occur due to the non-planar nature of the measured sample. Postprocessing algorithms can be implemented to correct this distortion. Here we present an OCT phantom designed to confirm the validity of post-processing algorithms used for measuring curved surfaces. A multi-purpose OCT phantom has been created within a fused silica plano-convex lens using the direct femtosecond laser writing technique. This phantom can be used to calibrate and quantitatively assess the performance (e.g. resolution, sensitivity and distortion) of OCT systems and associated post-processing algorithms for curved structures such as lenses. This novel OCT phantom has been characterized using an optical microscope and OCT systems.
\end{abstract}

Keywords: optical coherence tomography, OCT image distortion, femtosecond laser direct writing, OCT phantoms, OCT phantom fabrication

\section{INTRODUCTION}

Optical coherence tomography (OCT) systems can obtain non-invasive, cross-sectional, high-resolution, real-time, invivo images and were initially demonstrated by Huang et al. in 1991 [1]. The core of the OCT system is a low-coherence interferometer, which can probe the change in refractive index with depth of a transparent sample. In recent years, OCT systems have become widely used in the medical field (e.g. in ophthalmology) to assist with the diagnosis of diseases such as glaucoma. However, there is no ubiquitous standard that can be used to calibrate and assess the performance of different OCT systems and there is a requirement for multi-purpose OCT phantoms to act as a three-dimensional optical ruler.

Previously, various methods of making OCT phantoms have been presented. Woolliams et al. proposed a solid point spread function (PSF) phantom made of resin doped with $400 \mathrm{~nm}$ diameter nanoparticles with an even spread of 300$800 \mathrm{~nm}$ by mixing using an overhead stirrer [2]. Similarly, Agrawal et al. created a PSF phantom by embedding nanoparticles (i.e. gold-silica nanoshells) into an optically transparent medium, with resultant phantoms exhibiting a submicrometer spatial resolution [3]. However, the results showed some uncertainty due to the occasional aggregation of the particles and also the imperfections of the silicone. The fabrication process involves multiple steps which could introduce additional uncertainty. Tomlins et al. demonstrated the use of a femtosecond laser machining technique to fabricate planar OCT phantoms and this method enables accurate positioning of the defects and shows the possibility of a highly repeatable fabrication process [4]. This work produced a series of test patterns written in 3 dimensions in a planar fused silica substrate, and has been adopted commercially by Arden Photonics Ltd., Solihull, UK [5]. Sandrian et al. presented a method using a combination of femtosecond laser inscription and nanoparticle embedded resin to fabricate a 3D imaging calibration phantom [6]. Here, an automated assessment of 3D PSF pattern is used to evaluate the OCT system performance [6]. However, one disadvantage of this fabrication method is that the random dispersion of the nanoparticles is not repeatable, with each phantom being unique [6]. Moreover, the phantoms are limited in terms of the inscription depth due to the use of a high numerical aperture (NA) inscription lens to achieve micro-scale defects [6].

*Email: luy12@aston.ac.uk; Phone: +44 0121204 3616;

Advanced Fabrication Technologies for Micro/Nano Optics and Photonics XI, edited by Georg von Freymann,

Winston V. Schoenfeld, Raymond C. Rumpf, Proc. of SPIE Vol. 10544, 105441B · @ 2018 SPIE

CCC code: $0277-786 \mathrm{X} / 18 / \$ 18 \cdot$ doi: $10.1117 / 12.2290422$ 
More recently, Diaz et al. presented an inverse pyramidal structure for 3D geometrical calibration using a deep reactive ion etching method [7]. The fabrication method requires manual processes such as stacking and positioning and for OCT calibration an additional lens needs to be added to see the distortion effect which could introduce additional alignment issues [7]. Moreover, all these fabrication methods result in planar phantoms that do not fulfil the requirements of those systems designed for measuring curved structures such as contact lenses. Coldrick et al. demonstrated the measurement capability of using an OCT system (the Optimec is 830 system) to measure and inspect curved surfaces (i.e. contact lenses), however, the system would further benefit from a large-area calibration method [8].

To address this and building on the previous work from [4], a novel OCT calibration phantom has been inscribed in a plano-convex lens using direct femtosecond laser writing technique. The phantom can simulate the curvature of a contact lens and be used to test the validity of post-processing techniques. The use of a plano-convex lens (rather than a convex lens) is important because it allows the calibration pattern to be written through the non-distorting flat face of the lens and then viewed/used through the distorting curved face.

\section{EXPERIMENTAL SETUP}

The phantom was directly inscribed below the plane surface of a plano-convex lens (LA4380, Thorlabs), which is made of fused silica with a diameter of $25 \mathrm{~mm}$, a radius of curvature of $46 \mathrm{~mm}$, central thickness of $3.8 \mathrm{~mm}$ and an edge thickness of $2.0 \mathrm{~mm}$.

To fabricate the device a laser micromachining system (built by Oxford Lasers Ltd, UK) was used. The system contains a femtosecond laser source (Amplitude Systemes s-Pulse HP) that uses Ytterbium as the active laser medium and exhibits a central wavelength of $1026 \mathrm{~nm}$, pulse duration of $\sim 500 \mathrm{fs}$ and is set at a repetition rate of $100 \mathrm{kHz}$. The inscription lens has a magnification of 100x (Mititoyo M Plan Apo NIR Series) with a numerical aperture (NA) of 0.5 which results in a laser spot size of approximately $2.5 \mu \mathrm{m}$ at the focus. The sample is mounted on a software-based motion control system (Aerotech A3200). A goniometer located under the sample allows for precise manual position and angle control of the sample in the initial alignment process. The laser system layout is shown in Figure 1 below.

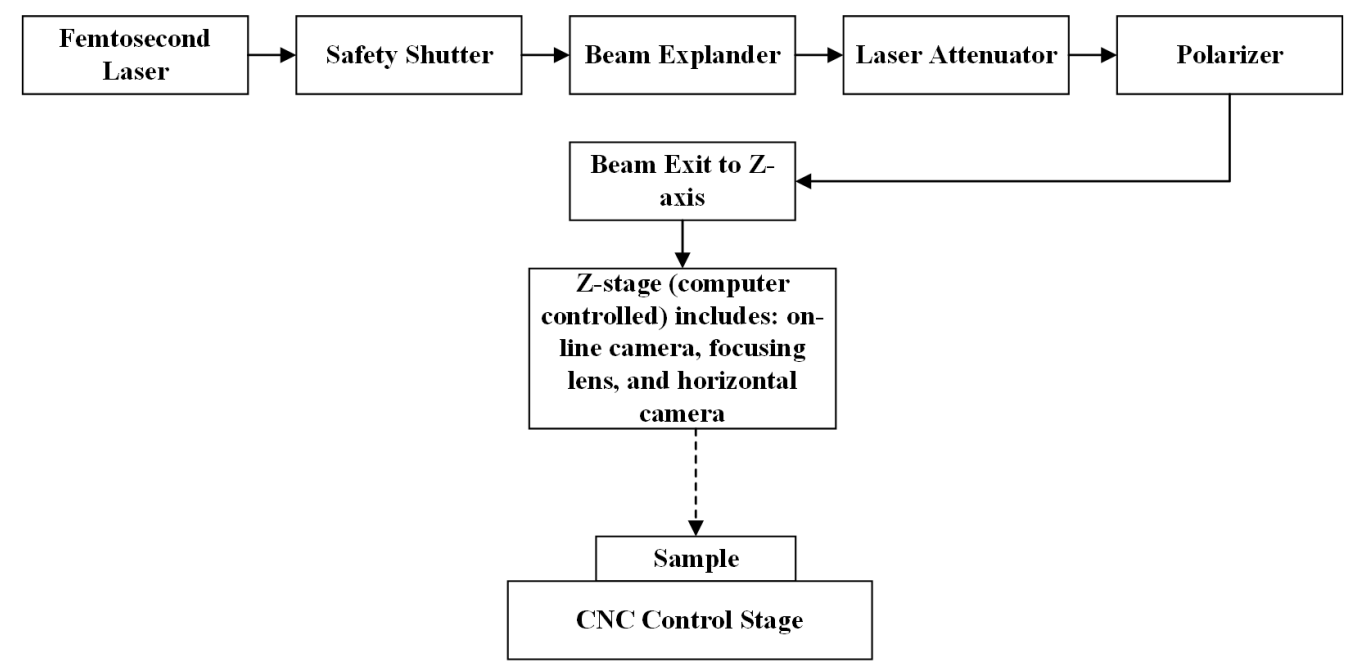

Figure 1. Schematic diagram of the laser system set-up at a $1026 \mathrm{~nm}$ central wavelength with a repetition rate of $100 \mathrm{kHz}$ and a 100x inscription lens.

The optical phantoms were characterized using both optical microscopy (Axioscope 2 MOT Plus, Zeiss) and two different spectral-domain OCT (SD-OCT) systems (Optimec is830 and Thorlabs Ganymede series). With the optical microscope, both the $5 \mathrm{x}$ objective lens and the $20 \mathrm{x}$ objective lens were used for the measurement. The two OCT systems have different resolutions for comparison and are detailed in Table 1. 
Table 1. Parameters of the two OCT systems used

\begin{tabular}{|l|c|c|}
\hline OCT system model & Optimec is 830 SD-OCT & Thorlabs Ganymede Series SD-OCT \\
\hline Central wavelength & $830 \mathrm{~nm}$ & $930 \mathrm{~nm}$ \\
\hline Axial resolution & $12 \mu \mathrm{m}($ air) & $5.8 \mu \mathrm{m}$ (air) \\
\hline Lateral resolution & $30 \mu \mathrm{m}$ & $8 \mu \mathrm{m}$ \\
\hline Sensitivity & $107 \mathrm{~dB}($ at $1.2 \mathrm{kHz})$ & $101 \mathrm{~dB}($ at $5.5 \mathrm{kHz})$ \\
\hline
\end{tabular}

\section{OCT PHANTOM DESIGN AND FABRICATION}

A grid-like phantom was inscribed on a plano-convex lens as shown in Figure 2 using a direct femtosecond laser writing technique. A non-linear multi-photon absorption process occurs when there is an interaction between the focused laser beam and the material, which induces refractive index changes. The average power of the laser was set to $188 \mathrm{~mW}$ (i.e. $20 \%$ of the total energy level) which results in $1880 \mathrm{~nJ}$ per pulse and therefore, a peak pulse power of $3760 \mathrm{~kW}$.

The inscribed pattern consisted of a series of $\mathrm{X}$ and $\mathrm{Y}$ lines that make a $100 \mu \mathrm{m}$ grid in a plane parallel to the flat surface of the lens. The size of the inscription was set to $25 \mathrm{~mm} \times 25 \mathrm{~mm}$ in order to cover the entire lens area. The first grid was inscribed $150 \mu \mathrm{m}$ in apparent depth (AD) below the flat side of the lens which has a real depth (RD) of $219.15 \mu \mathrm{m}$ calculated as $150 \mu \mathrm{m} \times 1.461$ (fused silica refractive index). The sample was then moved $50 \mu \mathrm{m}$ (in AD) in the $\mathrm{Z}$ direction and the grid pattern replicated and this process was repeated until there were 8 layers of $100 \mu \mathrm{m}$ grid patterns in total. To avoid scattering from pre-written lines, the inscription process starts from the lowest layer which is located at $500 \mu \mathrm{m}$ in the apparent depth (AD) below the surface or $730 \mu \mathrm{m}$ below the surface in the real depth (RD). This process enables each layer to be clearly inscribed.

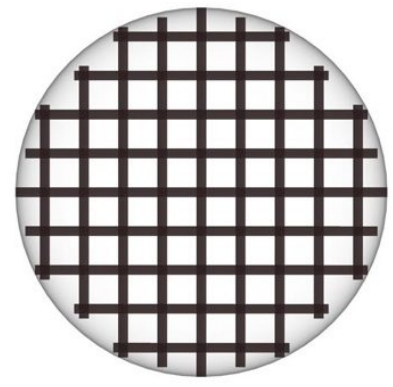

(a)

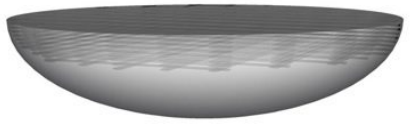

(b)

Figure 2. Conceptual drawings of the OCT phantom pattern: (a) the view from the top; (b) the view from the side.

Alignment is a big challenge when placing the sample on the CNC stage of the laser system, due to the difficulty in determining whether the planar side of the lens was parallel to the CNC stage. To overcome this challenge, three equidistant points close to the edge of the lens were focused upon to ensure the lens was flat. An alignment validation program performed a series of inscriptions to verify the alignment, ensuring that the flat side of the lens was parallel to the XY axis of the translation stage. Minor adjustments were then made on the sample's alignment using both software control and the manual adjustments of a goniometer stage. The process was repeated until the surface was suitably flat.

\section{RESULTS AND DISCUSSION}

The phantom was characterized first using an optical microscope (Axioscope 2 MOT Plus, Zeiss). Example microscope images are shown in Figure 3. Figure 3(a) shows an image taken with a 5x objective lens from the planar side of the phantom, and Figure 3(b) is a close-up of this using a 20x objective lens and gives the dimensions of the cell under 
inspection showing a cell size of $\sim 100 \mu \mathrm{m} \times 100 \mu \mathrm{m}$. Figure 3(c) shows the image taken from the curved surface and which shows the defocusing of the grid towards the outer edges of the image due to the effect of the lens curvature.

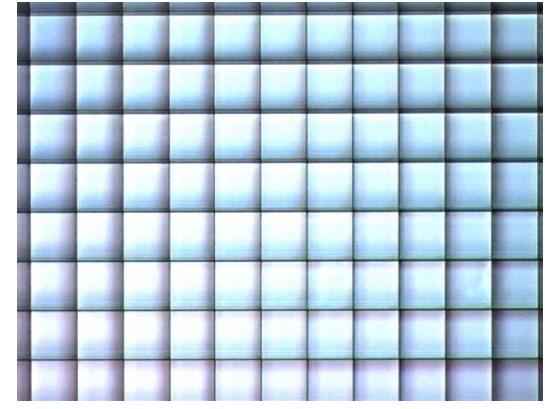

(a)

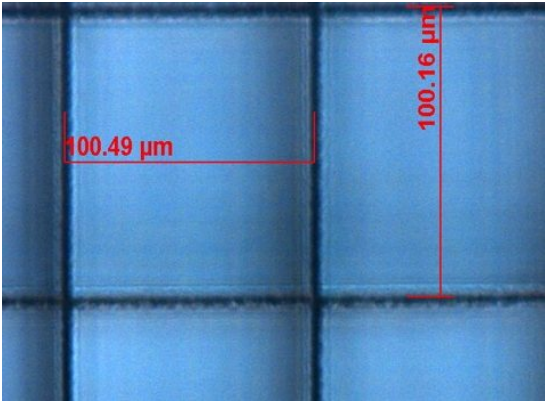

(b)

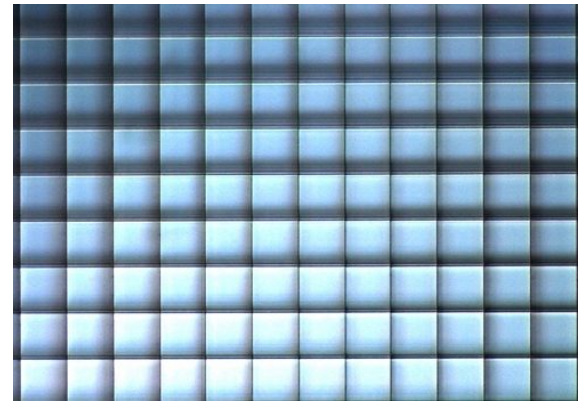

(c)

Figure 3. (a) Microscope image using a 5x objective lens from the planar side of the phantom, (b) the OCT phantom grid pattern cell size measurement using a $20 \mathrm{x}$ objective lens and giving the cell size of $\sim 100 \mu \mathrm{m} \times 100 \mu \mathrm{m}$, (c) the view from the curved side of the phantom.

A comparison between design parameters and measured values is given in Figure 4. The design parameters and the measured values closely match each other, which verifies the accuracy of fabrication process.

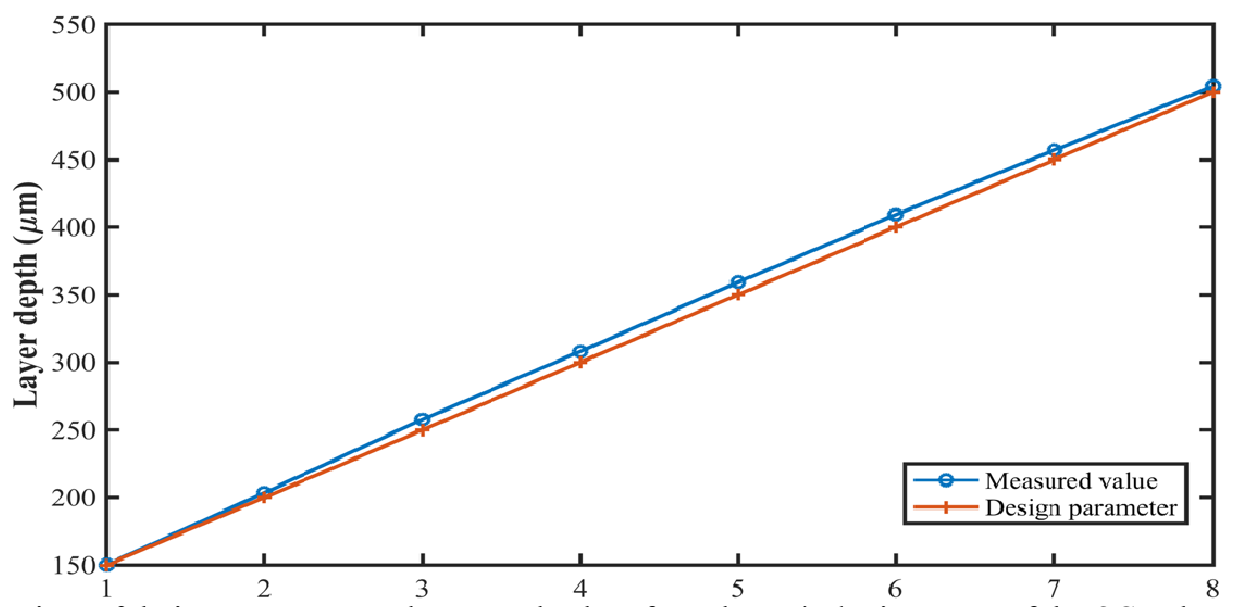

Figure 4. Comparison of design parameters and measured values from the optical microscope of the OCT phantom design. Measurements are all based on apparent depth (AD).

Subsequent to microscope based measurements, the phantom was characterized using the two SD-OCT systems. The images taken from those two OCT systems are shown in Figure 5(a) and (b). Both OCT images consistently match the design parameters and microscope measurements (the OCT image data was measured in pixels and converted back to a micrometer scale).

The Thorlabs Ganymede OCT system has a better axial resolution of $5.8 \mu \mathrm{m}$ (in air) (the image scaling bar in Figure 5(b) is $100 \mu \mathrm{m}$ ) and yields a more precise measurement than the Optimec is 830 OCT system, which has an axial resolution of $12 \mu \mathrm{m}$ (in air) (the image scaling bar in Figure 5(a) is $500 \mu \mathrm{m}$ ). However, the Optimec is 830 provides a larger field of view and has a better sensitivity which could provide a higher contrast image. The summarized measurements from both OCT systems are listed in Table 2. These images were taken from the planar side of the OCT phantom. 


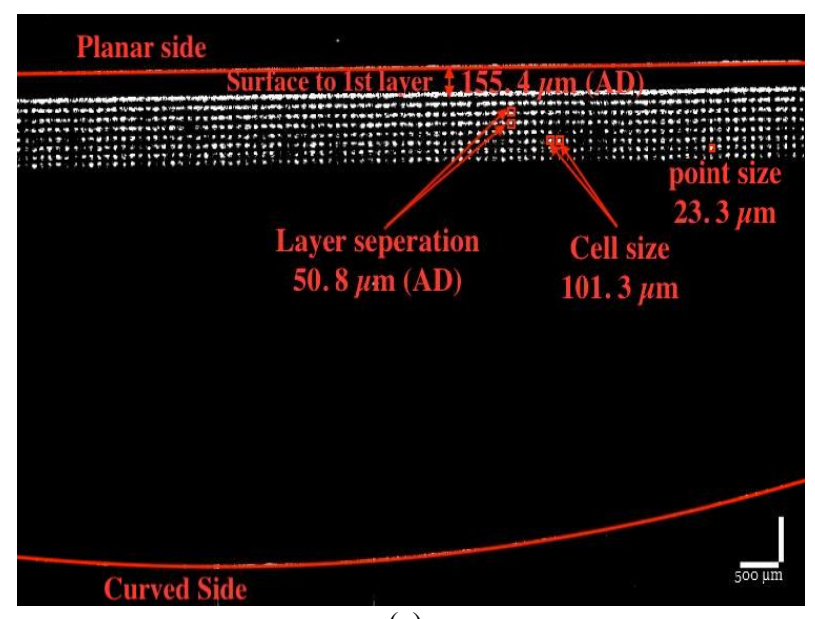

(a)

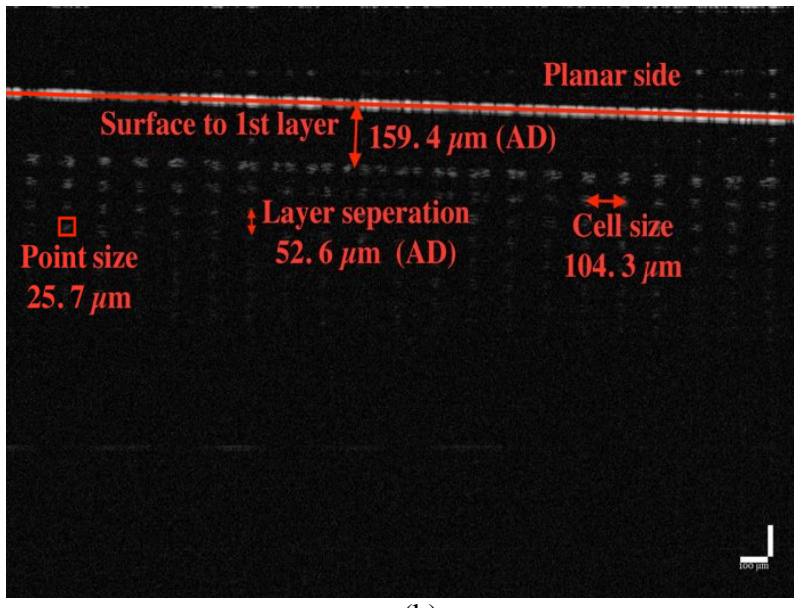

(b)

Figure 5. OCT images obtained from (a) the Optimec is 830 system with a scan size of $\sim 12 \mathrm{~mm} \times 5.6 \mathrm{~mm}$ and (b) Thorlabs Ganymede SD-OCT system with a scan size of $\sim 4 \mathrm{~mm} \times 1.9 \mathrm{~mm}$ for comparison with design parameters and between OCT systems with annotations.

Table 2. OCT system characterization and comparison of OCT systems results

\begin{tabular}{|l|c|c|}
\hline OCT system model & Optimec is $830 \mathrm{SD}-\mathrm{OCT}$ & Thorlabs Ganymede SD-OCT \\
\hline Image scaling & $500 \mu \mathrm{m}$ & $100 \mu \mathrm{m}$ \\
\hline Surface to 1st layer (AD) & $155.4 \mu \mathrm{m}$ & $159.4 \mu \mathrm{m}$ \\
\hline Layer separation (AD) & $50.8 \mu \mathrm{m}$ & $52.6 \mu \mathrm{m}$ \\
\hline Grid cell size & $101.3 \mu \mathrm{m}$ & $104.3 \mu \mathrm{m}$ \\
\hline
\end{tabular}

Figure 6 and Figure 7 show the OCT images of the phantom taken from the curved side of the lens. It can be seen that cross section of the grid lines looks wider. Using images from both sides of the lens could be used to confirm the validity of the post-processing algorithms since the pattern should look the same in both directions.

Figure 6 was acquired using the Thorlabs Ganymede OCT system. The cross-section of the grid pattern is expanded due to the curvature of the lens, and the point size measured in this distorted image appears to be $\sim 47.8 \mu \mathrm{m}$. This is approximately 2 times as large as the non-distorted image which was determined from Figure 5 (a) to be $\sim 23.3 \mu \mathrm{m}$.

In Figure 7, taken with the Optimec is 830 system, it can be seen that the cross section of the grid and the planar surface at the bottom of the image appears to be bending. This shows that the distortion from the curved surface is present and therefore suitable post processing of the image is required to correct for such distortion. 


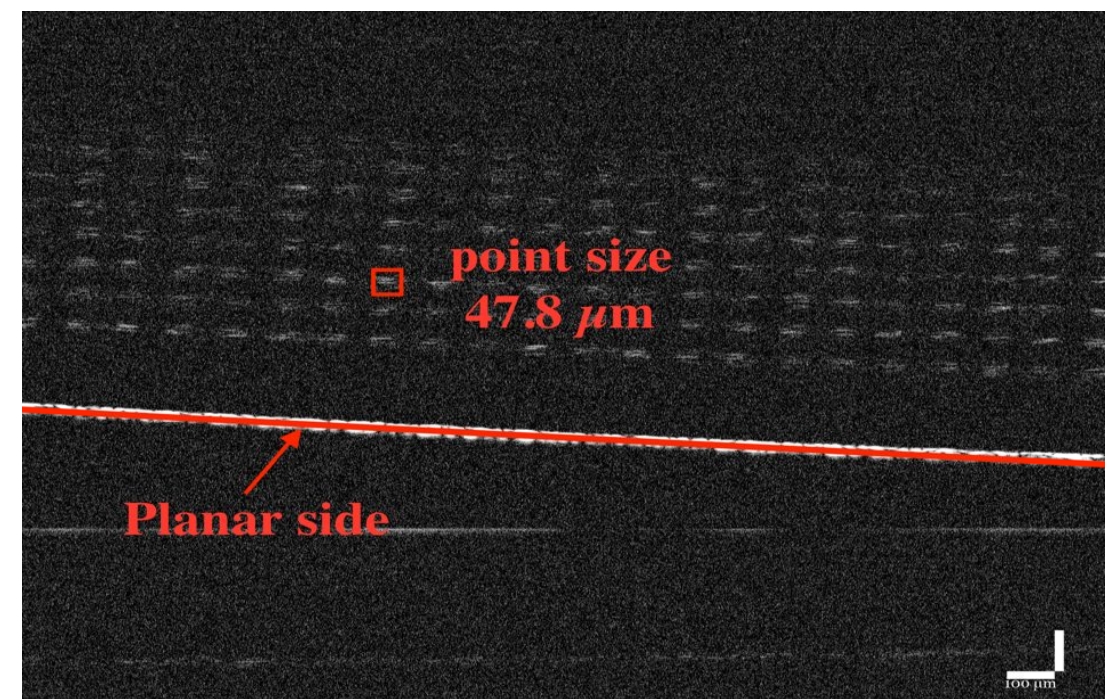

Figure 6. OCT image taken from the curved side (by using the Thorlabs Ganymede SD-OCT with a central wavelength of $930 \mathrm{~nm}$ and an axial resolution of $5.8 \mu \mathrm{m})$

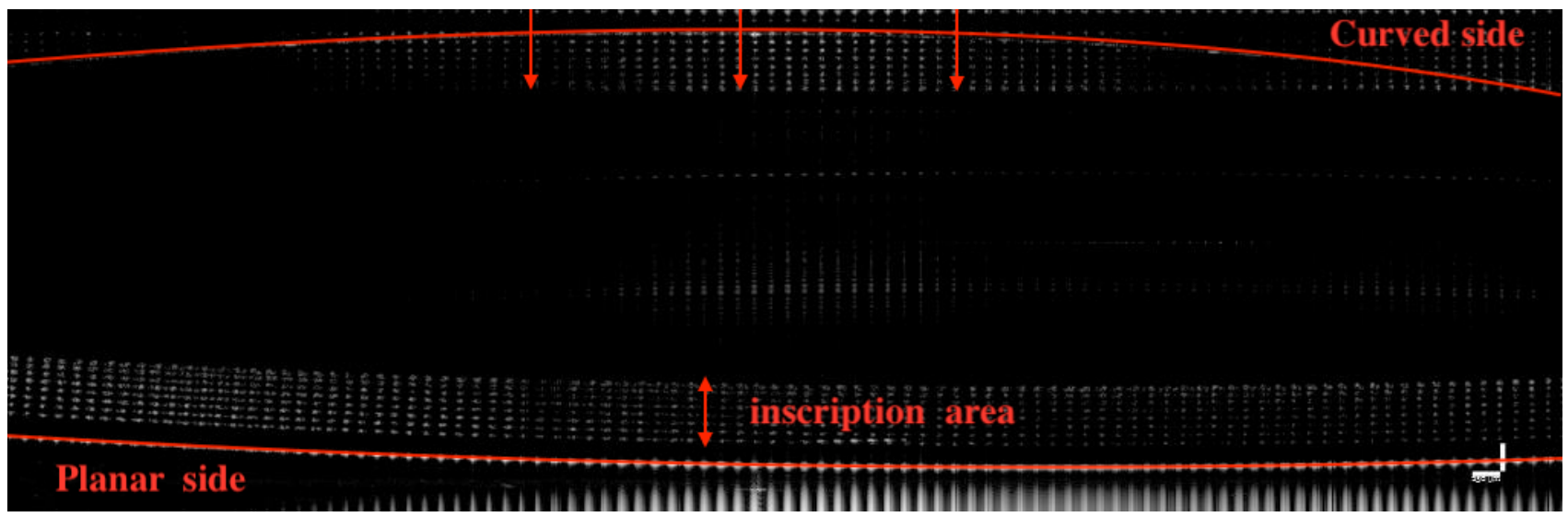

Figure 7. OCT image taken from the curved side (by using the Optimec is 830 system with a central wavelength of $830 \mathrm{~nm}$ and an axial resolution of $12 \mu \mathrm{m})$

\section{CONCLUSIONS}

For the first time, an OCT phantom has been successfully inscribed in a plano-convex lens and characterized using an optical microscope and OCT systems. This work may help in overcoming the difficulties of validating the postprocessing algorithms used in OCT applications such as ophthalmology and contact lens metrology that are required due to the focusing or defocusing effects from the curved surfaces. This distortion is also an issue for the fabrication of phantoms and in this case, it is overcome by inscribing the calibration grid from the planar side of the lens.

This OCT phantom design approach has potential applications in the field of OCT systems and medicine (i.e. ophthalmology). It can be accurately reproduced as an optical calibration device. It can be used to validate the postprocessing algorithms of the OCT system to see whether it can resolve distortion introduced by curved surfaces, in addition to providing a check for current systems and for new systems after assembly. The plano-convex lens can also serve a dual purpose as the planar side shows the non-distorted image and the curved side can be used to check whether the image is distorted or has been fixed through the post-processing procedure. Currently, software based upon quantitative methods is under the development and various types of OCT systems need to be tested. 


\section{ACKNOWLEGEMENTS}

We would like to acknowledge the sponsorship of MKS Instruments, Inc. for the SPIE Student Author Travel Grant Award and Arden Photonics Ltd. and the School of Engineering and Applied Science at Aston University for the sponsorship of Yang Lu's PhD.

\section{REFERENCES}

[1] Huang, D., Swanson, E. A., Lin, C. P., Schuman, J. S., Stinson, W. G., Chang, W., Hee, M. R., Flotte, T., Gregory, K., Puliafito, C. A., and Fujimoto, J. G., "Optical Coherence Tomography," Science 254(5035), 11781181 (1991).

[2] Woolliams, P. D., Ferguson, R. A., Hart, C., Grimwood, A., and Tomlins, P. H., "Spatially deconvolved optical coherence tomography," Applied Optics 49(11), 2014-2021 (2010).

[3] Agrawal, A., Pfefer, T. J., Gilani, N., and Drezek, R., "Three-dimensional characterization of optical coherence tomography point spread functions with a nanoparticle-embedded phantom," Optics Letters 35(13), 2269-2271 (2010).

[4] Tomlins, P. H., Smith, G. N., Woolliams, P. D., Rasakanthan, J., and Sugden, K., "Femtosecond laser microinscription of optical coherence tomography resolution test artifacts," Biomedical Optics Express 2(5), 13191327 (2011).

[5] Arden Photonics, "Optical Coherence Tomography APL-OP01," < $\underline{\text { http://www.ardenphotonics.com/product- }}$ types/oct/> (20 December 2017).

[6] Sandrian, M. G., Tomlins, P., Woolliams, P., Rasakanthan, J., Lee, G. C. B., Yang, A., Povazay, B., Alex, A., Sugden, K., and Drexler, W., "Three-dimensional calibration targets for optical coherence tomography," Proceedings of SPIE 822914 (2012).

[7] Díaz, J. D., Stritzel, J., Rahlves, M., Majdani, O., Reithmeier, E., Ortmaier, T., and Roth, B., "One step geometrical calibration method for optical coherence tomography," Journal of Optics 18(1), 015301 (2016).

[8] Coldrick, B. J., Richards, C., Sugden, K., Wolffsohn, J. S., and Drew, T. E., "Developments in contact lens measurement: A comparative study of industry standard geometric inspection and optical coherence tomography," Contact Lens and Anterior Eye 39(4), 270-276 (2016). 